\title{
Controle químico de Anastrepha fraterculus (Wied.) (Diptera: Tephritidae) em laboratório
}

\author{
Chemical control of Anastrepha fraterculus (Wied.) \\ (Diptera: Tephritidae) in laboratory
}

\section{Priscila Lang Scoz ${ }^{1}$ Marcos Botton ${ }^{2}$ Mauro Silveira Garcia ${ }^{3}$}

\section{RESUMO}

\begin{abstract}
$O$ efeito de quatro novos grupos químicos de inseticidas incluindo avermectina (benzoato de emamectina), éter piretróide (etofemprox), neoniconitnóide (imidacloprid, thiacloprid e thiamethoxan) e naturalyte (spinosad) foram avaliados em laboratório $\left(25 \pm 3^{\circ} \mathrm{C}\right.$, umidade relativa de $70 \pm$ $10 \%$ e fotofase de 12 horas), visando ao controle de adultos $e$ ovos/larvas de Anastrepha fraterculus comparando-os com os fosforados fenthion e thrichlorphon. O benzoato de emamectina não foi eficiente no controle de adultos de A. fraterculus via contato $e$ ingestão. O etofenprox, imidacloprid, spinosad $e$ thiamethoxan foram eficientes no controle de adultos de $\boldsymbol{A}$. fraterculus via contato e ingestão, proporcionando maior mortalidade via ingestão. Os novos inseticidas não provocaram mortalidade significativa de ovos/larvas de A. fraterculus localizados no interior de maçãs, enquanto que os fosforados fenthion e thrichlorphon resultaram em $100 \%$ de mortalidade das fases imaturas e adultos. Os novos inseticidas apresentam potencial para uso nas iscas tóxicas, substituindo os fosforados para o controle de adultos.
\end{abstract}

Palavras-chave : mosca-das-frutas sul americana, isca-tóxica, neonicotinoides.

\section{ABSTRACT}

The South American Fruit Fly, Anastrepha fraterculus is one of the most important pest of temperate fruit crops. The effect of four new inseticide groups to replace organophosphate compounds for A. fraterculus control was evaluated under laboratory conditions $\left(25 \pm 3^{\circ} \mathrm{C}\right.$, relative humity of $70 \pm 10 \%$ and 12:12 L:D). Emamectin benzoate, etofenprox, imidacloprid, spinosad, thiacloprid and thiamethoxan were evaluated to control adults by contact and ingestion and against eggs/larvae inside apple fruits compared with fenthion and thrichlorphon. Emamectin benzoate was not efficient to control adults of A. fraterculus by contact and ingestion. Etofenprox, imidacloprid, spinosad and thiamethoxan were efficient to control adults by contact and ingestion being more toxic by ingestion. No new insecticide controlled eggs/larvae inside apple fruit while organophosphate compounds caused $100 \%$ of mortality of immature stages and adults. The new insecticides showed more potential to substitute organophosphate compounds in bait sprays for adult control.

Key words: south american fruit fly, neonicotinoids, toxic bait.

\section{INTRODUÇÃO}

Entre os principais problemas associados à produção de frutas de clima temperado, destacamse os insetos-praga, sendo que as moscas-das-frutas são responsáveis por danos elevados em diferentes culturas (CALKINS \& MALAVASI, 1995; SALLES, 1995a). Na região sul do Brasil, Anatrepha fraterculus (Wied.) (Diptera: Tephritidae) representa mais de $86 \%$ das espécies do gênero capturadas nos pomares, sendo a principal responsável por prejuízos às fruteiras temperadas (BLEICHER et al., 1982; SALLES, 1995a; NORA et al., 2000).

O controle de $A$. fraterculus tem como base a aplicação de iscas tóxicas e pulverizações de inseticidas fosforados em cobertura que controlam adultos e ovos/larvas no interior dos frutos (SALLES \& KOVALESKI, 1990; REIS FILHO, 1994;

\footnotetext{
${ }^{1}$ Engenheiro Agrônomo, Mestre em Fitossanidade, Universidade Federal de Pelotas (UFPel). CP 354, 96010-900, Pelotas, RS.

${ }^{2}$ Engenheiro Agrônomo, Pequisador, Doutor em Entomologia, EMBRAPA Uva e Vinho, CP 130, 95700-000, Bento Gonçalves, RS, Email: marcos@cnpuv.embrapa.br. Autor para correspondência

${ }^{3}$ Engenheiro Agrônomo, Professor, Doutor em Entomologia, UFPel.
} 
CALKINS \& MALAVASI, 1995; SALLES, 1995a; KOVALESKI et al., 2000; KOVALESKI \& RIBEIRO, 2003). Este método de controle tem sido eficaz por mais de 40 anos, sem haver relatos de seleção de populações mais resistentes (PUZZI \& ORLANDO, 1957; KOVALESKI et al., 2000). Os inseticidas fosforados, entretanto, caracterizam-se por apresentar elevada toxicidade, baixa seletividade aos inimigos naturais e grande período de carência (SALLES, 1998; KOVALESKI \& RIBEIRO, 2003), levando a uma preocupação crescente sobre os efeitos dos resíduos nos alimentos e no ambiente. Por estes motivos, o registro dos fosforados está sendo revisto nos Estados Unidos da América através do "Ato de Proteção à Qualidade dos Alimentos" (Food Quality Protection Act), de 1996 (EPA, 2003) e no Brasil, através das limitações conferidas pelos Sistemas de Produção Integrada de Frutas.

A redução das alternativas para o controle do inseto leva à necessidade de pesquisar novos produtos, principalmente para o período de précolheita quando a baixa carência dos inseticidas é fundamental. Isso é ainda mais urgente devido à tendência mundial de emprego de sistemas de produção que exigem, além da qualidade externa das frutas, controle sobre todo o sistema, incluindo a análise de resíduos nas frutas (FACHINELLO, 2003). Este trabalho foi conduzido com o objetivo de avaliar, em laboratório, o efeito de novos grupos químicos de inseticidas como possíveis substitutos aos fosforados para o controle de A. fraterculus.

\section{MATERIAL E MÉTODOS}

Os experimentos foram conduzidos com insetos provenientes da criação mantida no Laboratório de Entomologia, da Embrapa Uva e Vinho em Bento Gonçalves, RS, utilizando mamão papaia como substrato para o desenvolvimento larval (CRUZ et al., 1994). Todos os experimentos foram conduzidos na temperatura de $25 \pm 3^{\circ} \mathrm{C}$, umidade relativa (UR) de $70 \pm 10 \%$ e fotofase de 12 horas.

Os inseticidas (grupos químicos) e doses avaliadas foram: a) benzoato de emamectina (avermectina) a 0,$75 ; 1,0$ e $1,25 \mathrm{~g} 100 \mathrm{~L}^{-1}$ de água (Proclain $5 \mathrm{SG}$ a 15, 20 e 25g $100 \mathrm{~L}^{-1}$ ), b) etofenprox

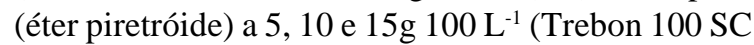

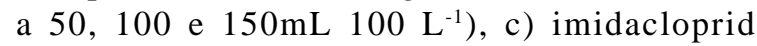
(neonicotinóide) a 8,10 e $12 \mathrm{~g} 100 \mathrm{~L}^{-1}$ (Provado 200

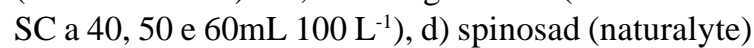
a 4,8; 7,2 e 9,6g $100 \mathrm{~L}^{-1}$ (Tracer $480 \mathrm{SC}$ a 10,15 e 20 $\mathrm{mL} 100 \mathrm{~L}^{-1}$ ), e) thiacloprid (neonicotinóide) a 48, 96

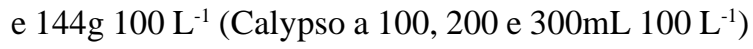

e f) thiamethoxan (neonicotinóide) a10, 12,5 e 15g $100 \mathrm{~L}^{-1}$ (Actara $250 \mathrm{WG}$ a 40, 50 e $60 \mathrm{~g} 100 \mathrm{~L}^{-1}$ ). Como padrão de controle foi usado os fosforados thrichlorphon a $150 \mathrm{~g} 100 \mathrm{~L}^{-1}$ (Dipterex $500 \mathrm{SC}$ a 300 $\mathrm{mL} 100 \mathrm{~L}^{-1}$ ) e o fenthion a $50 \mathrm{~g} 100 \mathrm{~L}^{-1}$ (Lebaycid 500

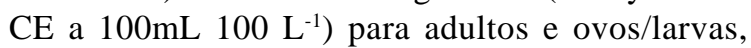
respectivamente, comparando-os com uma testemunha tratada com água destilada. As doses dos novos inseticidas foram definidas em experimentos preliminares, onde a maior dose provocou mortalidade acima de $90 \%$ dos adultos por contato. No caso dos inseticidas, onde não se obteve incrementos significativos de mortalidade com o aumento na quantidade de produto aplicada, as doses avaliadas foram definidas com base nas quantidades empregadas para outras pragas associadas às fruteiras temperadas.

O bioensaio para avaliar o controle dos adultos pelos inseticidas via ação de contato foi conduzido no delineamento inteiramente casualizado, utilizando-se dez repetições por tratamento, sendo que cada unidade experimental foi formada por dois casais com idade de 10 a 14 dias. Estes foram retirados das gaiolas de criação com auxílio de um tubo de ensaio $(10 \mathrm{~cm})$ e colocados num saco plástico $(20 \mathrm{~cm} \times 30 \mathrm{~cm})$, onde foram anestesiados com $\mathrm{CO}_{2}$ por 30 segundos. Os insetos anestesiados foram dispostos numa placa de Petri (10cm de diâmetro) e submetidos à aplicação tópica em torre de pulverização (Burkard Scientific Uxbridge UK), a pressão de $10 \mathrm{lb} \mathrm{pol}^{-2}$, utilizando-se $1 \mathrm{~mL}$ de calda por aplicação, obtendo-se uma deposição média de resíduo úmido de $1,8 \mathrm{mg} \mathrm{cm}^{-2}$. Após a aplicação, os casais foram colocados em gaiolas confeccionadas a partir de copo plástico transparente $(5 \mathrm{~cm}$ de diâmetro x $10 \mathrm{~cm}$ de altura), que tiveram o fundo retirado e substituído por tecido voil, permitindo a troca de gases com o ambiente. Como alimento, foi oferecido solução de mel a 2,5\% através de um rolete de algodão, num frasco de vidro de $10 \mathrm{~mL}$.

O bioensaio, para avaliar a ação dos inseticidas por ingestão, foi conduzido com metodologia similar ao de contato. Os inseticidas foram oferecidos numa solução/suspensão aquosa associada à mel a $2,5 \%$, através de um rolete de algodão inserido em um tubo de $10 \mathrm{~mL}$. Após o preparo das soluções/suspensões, foi adicionado $1 \mathrm{~g} \mathrm{~L}^{-1} \mathrm{de}$ corante vermelho Ponceau (Sigma Chemical Co.) para atuar como indicador do consumo do inseticida, observado através da cor vermelha no abdômen dos insetos (CRUZ et al., 1997).

$\mathrm{O}$ número de insetos sobreviventes, nos dois experimentos, foi avaliado 24 e 72 horas após o tratamento (HAT) com os inseticidas. Este intervalo 
de avaliação foi definido nos experimentos preliminares, demonstrando o efeito de choque (24 HAT) e o tempo máximo em que não ocorreram incrementos significativos de mortalidade para todos os produtos (72 HAT).

O bioensaio, visando ao controle de ovos e larvas por ação de profundidade, foi realizado com maçãs da cultivar Gala, com cinco centímetros de diâmetro, oferecidas a fêmeas de $\boldsymbol{A}$. fraterculus (30 dias de idade) para oviposição, no interior de gaiolas de criação $(25 \times 25 \times 25 \mathrm{~cm})$, por 24 horas. Após este período, os frutos retirados das gaiolas foram acondicionados em copos plásticos $(300 \mathrm{~mL})$, cobertos por tecido voil por três dias, tempo suficiente para ocorrer a eclosão das larvas (SALLES, 2000). Passado esse período, os frutos foram imersos em $1 \mathrm{~L}$ da calda inseticida, por 30 segundos, na maior dose de cada inseticida avaliado no bioensaio de contato. Após o tratamento, os frutos foram deixados à temperatura ambiente, por duas horas, para secagem, sendo acondicionados individualmente em copo plástico de $300 \mathrm{~mL}$ fechado com tecido voil.

$\mathrm{O}$ experimento foi conduzido no delineamento experimental inteiramente casualizado com dez repetições (um fruto por repetição) por tratamento. O número de larvas vivas por fruto foi avaliado quinze dias após a oviposição.

O número de insetos sobreviventes foi submetido à análise de variância, sendo as médias transformadas em raiz quadrada de $(x+0,5)$, comparando-se as médias pelo teste de Tukey, em nível de $5 \%$ de probabilidade de erro, através do programa Sisvar versão 4.3 (FURTADO, 1993). A mortalidade foi calculada pela fórmula de ABBOTT (1925).

\section{RESULTADOS E DISCUSSÃO}

A avaliação dos inseticidas 24 horas após o tratamento (HAT), para o controle de adultos de $\boldsymbol{A}$. fraterculus por contato, revelou que apenas os

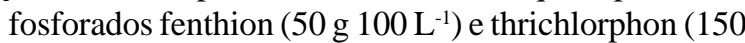
g.100 L-1), utilizados como padrão de comparação, provocaram $100 \%$ de mortalidade, demonstrando maior rapidez de controle que os novos inseticidas (Tabela 1). Resultados similares foram obtidos por SALLES \& KOVALESKI (1990) para o fenthion. No entanto, esses autores observaram apenas $16,7 \%$ de mortalidade de adultos com thrichlorphon, diferindo dos resultados obtidos neste trabalho. O etofenprox a $15 \mathrm{~g} 100 \mathrm{~L}^{-1}$ proporcionou mortalidade de $80 \%$ dos adultos, enquanto o mesmo inseticida na dose de $5 \mathrm{e}$ $10 \mathrm{~g} 100 \mathrm{~L}^{-1}$, imidacloprid a $12 \mathrm{~g} 100 \mathrm{~L}^{-1}$, spinosad a 9,6g $100 \mathrm{~L}^{-1}$ e thiamethoxan a 12,5 e $15 \mathrm{~g} 100 \mathrm{~L}^{-1}$ apresentam mortalidade entre 55 e $73 \%$ (Tabela 1). O benzoato de emamectina e thiacloprid nas três doses avaliadas, imidacloprid a 8 e $10 \mathrm{~g} 100 \mathrm{~L}^{-1}$ e spinosad a $7,2 \mathrm{~g} 100 \mathrm{~L}^{-1}$, proporcionou mortalidade equivalente à testemunha (Tabela 1).

As 72 HAT, spinosad $\left(9,6 \mathrm{~g} 100 \mathrm{~L}^{-1}\right)$,

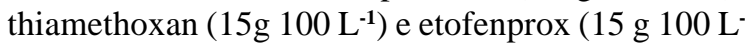
1) proporcionaram mortalidade próxima a $90 \%$, equivalendo-se aos fosforados. O imidacloprid $(12 \mathrm{~g}$ $100 \mathrm{~L}^{-1}$ ) resultou num controle próximo a $80 \%$ enquanto que o benzoato de emamectina, nas três doses, imidacloprid (8 e $\left.10 \mathrm{~g} 100 \mathrm{~L}^{-1}\right)$ e spinosad $(4,8 \mathrm{~g}$ $\left.100 \mathrm{~L}^{-1}\right)$ proporcionou uma mortalidade intermediária $(33<\% \mathrm{M}<63)$. O thiacloprid igualou-se a testemunha (Tabela 1).

No experimento de ingestão (Tabela 1), observou-se que os insetos ingeriram os produtos em todos os tratamentos, registrado pela cor vermelha do abdômen, devido à presença do corante na dieta. Este fato indica que os inseticidas não provocaram ação de repelência, e a mortalidade observada foi resultante da ação dos produtos. CRUZ et al. (1997) também observaram que dietas com fenthion, contendo o mesmo corante, não foram discriminadas por $\boldsymbol{A}$. fraterculus.

Da mesma forma que, no experimento de contato, os inseticidas fenthion $\left(50 \mathrm{~g} 100 \mathrm{~L}^{-1}\right) \mathrm{e}$ thrichlorphon $\left(150 \mathrm{~g} 100 \mathrm{~L}^{-1}\right)$ proporcionaram $100 \%$ de mortalidade 24 HAT, concordando com os resultados de SALLES \& KOVALESKI (1990) (Tabela 1). SALLES (1995b) também verificou que, seis horas após a exposição dos adultos de $\boldsymbol{A}$. fraterculus à isca com suco de pêssego (10\%) associada ao thrichlorphon

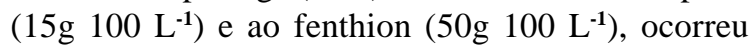
mortalidade dos adultos de 60 e $72 \%$, respectivamente.

Na primeira avaliação (24 HAT), não foi observada diferença significativa na mortalidade dos adultos entre os tratamentos com benzoato de emamectina $\left(0,75 ; 1,0\right.$ e $\left.1,25 \mathrm{~g} .100 \mathrm{~L}^{-1}\right)$, etofenprox (5g.100 L $\left.\mathrm{L}^{-1}\right)$, imidacloprid (8 e $\left.10 \mathrm{~g} 100 \mathrm{~L}^{-1}\right)$, spinosad

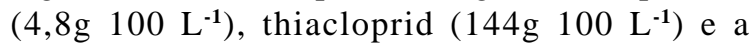
testemunha (Tabela 1). O etofenprox (10 e $15 \mathrm{~g} 100$ $\left.\mathrm{L}^{-1}\right)$, imidacloprid (12g $\left.100 \mathrm{~L}^{-1}\right)$, spinosad $(7,2$ e 9,6g

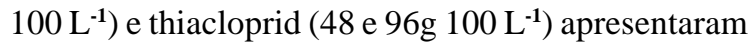
controle de A.fraterculus entre 40 e $60 \%$ (Tabela 1). Nesta avaliação, o thiamethoxan, nas três doses avaliadas, foi o único que apresentou mortalidade equivalente aos fosforados fenthion $\left(50 \mathrm{~g} 100 \mathrm{~L}^{-1}\right) \mathrm{e}$ thrichlorphon (150g $\left.100 \mathrm{~L}^{-1}\right)$.

As 72 HAT, o benzoato de emamectina manteve a baixa eficiência, inferior a $50 \%$, nas três doses avaliadas (Tabela 1). Os inseticidas etofenprox

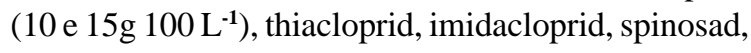

Ciência Rural, v.34, n.6, nov-dez, 2004. 
Tabela 1 - Número médio de insetos sobreviventes $(\mathrm{N} \pm \mathrm{EP})$ e mortalidade $(\mathrm{M})$ de adultos de Anastrepha fraterculus, 24 e 72 horas após o tratamento (HAT), via pulverização (contato) e ingestão. Temperatura $25 \pm 3^{\circ} \mathrm{C}$; UR: $70 \pm 10 \%$ e Fotofase: 12 horas.

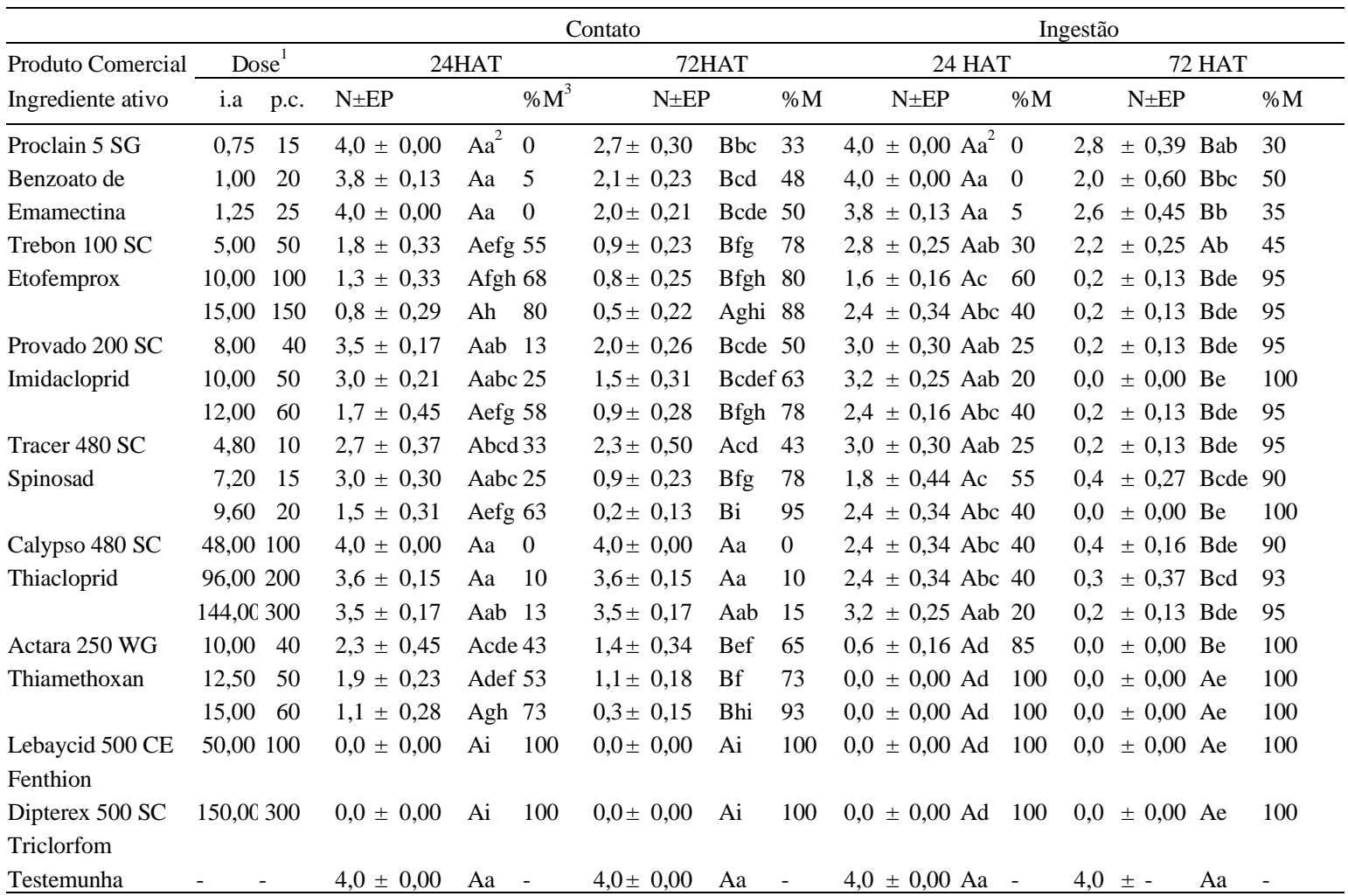

${ }^{1}$ Gramas ou $\mathrm{mL}$ de ingrediente ativo (i.a.) ou produto comercial (p.c.) por 100 litros de água.

${ }^{2}$ Médias seguidas por letras distintas maiúsculas na linha e minúsculas na coluna diferem entre si pelo teste de Tukey em nível de 5\% de probabilidade de erro.

${ }^{3}$ Percentagem de Mortalidade corrigida por Abbott (1925).

e thiamethoxan nas três doses avaliadas equivaleramse aos fosforados fenthion e thrichlorphon (Tabela 1). À exceção do benzoato de emamectina, os novos inseticidas foram mais eficientes no controle de adultos da mosca-das-frutas via ingestão do que por contato (Tabela 1). Este fato indica a possibilidade de emprego destes compostos como substitutos aos fosforados nas iscas tóxicas.

Os trabalhos com iscas tóxicas, utilizando novos grupos químicos, têm se concentrado no emprego do spinosad. KING \& HENNESEY (1996), avaliando uma isca que continha spinosad para o controle de $\boldsymbol{A}$. suspensa (Loew), não observaram repelência até a concentração máxima testada $(5 \mathrm{~g}$ $\left.100 \mathrm{~L}^{-1}\right)$. Os autores encontraram $100 \%$ de mortalidade nas doses de 2,7 e $0,59 \mathrm{~g} 100 \mathrm{~L}^{-1}$ após 24 HAT e 48 HAT, respectivamente, comentando que o spinosad é uma alternativa promissora para substituir o malathion $\left(190 \mathrm{~g} 100 \mathrm{~L}^{-1}\right)$ em programas de erradicação da espécie.
No experimento de ação por profundidade

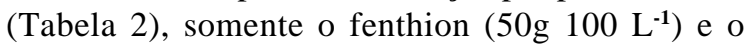
thrichlorphon $\left(150 \mathrm{~g} 100 \mathrm{~L}^{-1}\right)$ proporcionaram $100 \%$ de mortalidade de ovos/larvas de A. fraterculus. Os inseticidas benzoato de emamectina $\left(1,25 \mathrm{~g} 100 \mathrm{~L}^{-1}\right)$, etofenprox $\left(15 \mathrm{~g} 100 \mathrm{~L}^{-1}\right)$, imidacloprid $\left(12 \mathrm{~g} 100 \mathrm{~L}^{-1}\right)$, spinosad $\left(9,6 \mathrm{~g} 100 \mathrm{~L}^{-1}\right)$, thiacloprid $\left(144 \mathrm{~g} 100 \mathrm{~L}^{-1}\right) \mathrm{e}$ thiamethoxan $\left(15 \mathrm{~g} \quad 100 \quad \mathrm{~L}^{-1}\right)$ não reduziram significativamente $(\mathrm{p}<0,05)$ a população de larvas, equivalendo-se ao tratamento testemunha (Tabela 2).

A aplicação de fenthion $\left(50 \mathrm{~g} .100 \mathrm{~L}^{-1}\right) \mathrm{e}$ thrichlorphon $\left(150 \mathrm{~g} 100 \mathrm{~L}^{-1}\right)$ sobre frutos de ameixeira infestados por larvas de $\boldsymbol{A}$. fraterculus proporcionou resultados semelhantes a este experimento (SALLES \& KOVALESKI, 1990), bem como LORENZATO et al. (1986) e REIS FILHO (1994) verificaram que o fenthion $\left(50 \mathrm{~g} 100 \mathrm{~L}^{-1}\right)$, aplicado em cobertura na cultura da macieira, foi eficiente no controle do inseto.

Os adultos de A. fraterculus são altamente sensíveis à maioria dos inseticidas 
Tabela 2 - Número médio ( \pm EP) de larvas de Anastrepha fraterculus por fruto e porcentagem de mortalidade (\%M) proporcionado por inseticidas quinze dias após a oviposição em maçãs da cv. Gala em laboratório. Temperatura: $25 \pm 3^{\circ} \mathrm{C}$, UR: $70 \pm 10$, Fotofase de 12 horas.

\begin{tabular}{|c|c|c|c|c|c|c|c|c|}
\hline \multicolumn{2}{|c|}{ Inseticida } & \multicolumn{2}{|c|}{ Dose $^{1}$} & \multicolumn{4}{|c|}{$\mathrm{N} \pm \mathrm{EP}$} & \multirow[t]{2}{*}{$\% \mathrm{M}^{3}$} \\
\hline Ingrediente ativo & Produto comercial & i.a. & p.c. & & & & & \\
\hline Emamectina & Proclaim $5 \mathrm{SG}$ & 1,25 & 25 & 7,5 & \pm & 1,36 & $a^{2}$ & 0 \\
\hline Etofenprox & Trebon $100 \mathrm{SC}$ & 15,00 & 150 & 6,9 & \pm & 0,91 & $\mathrm{a}$ & 0 \\
\hline Imidacloprid & Provado $200 \mathrm{SC}$ & 12,00 & 60 & 7,4 & \pm & 1,48 & $\mathrm{a}$ & 0 \\
\hline Spinosad & Tracer $480 \mathrm{SC}$ & 9,60 & 20 & 7,3 & \pm & 0,79 & $\mathrm{a}$ & 0 \\
\hline Thiacloprid & Calypso 480 SC & 144,00 & 300 & 7,7 & \pm & 0,83 & $\mathrm{a}$ & 0 \\
\hline Thiamethoxan & Actara $250 \mathrm{WG}$ & 15,00 & 60 & 7,2 & \pm & 0,71 & $\mathrm{a}$ & 0 \\
\hline Fenthion & Lebaycid $500 \mathrm{CE}$ & 50,00 & 100 & 0,0 & \pm & 0,00 & $\mathrm{~b}$ & 100 \\
\hline Thichlorphon & Dipterex 500 SC & 150,00 & 300 & 0,0 & \pm & 0,00 & $\mathrm{~b}$ & 100 \\
\hline Testemunha & - & - & - & 7,1 & \pm & 0,96 & $\mathrm{a}$ & - \\
\hline
\end{tabular}

${ }^{1}$ Gramas ou $\mathrm{mL}$ de ingrediente ativo (i.a.) ou produto comercial (p.c.) por 100 litros de água.

${ }^{2}$ Médias seguidas por letras distintas na coluna diferem entre si pelo teste de Tukey em nível de 5\% de probabilidade de erro.

${ }^{3}$ Percentagem de mortalidade corrigida por Abbott (1925).

fosforados e piretróides, não havendo casos reportados de seleção de resistência (CRUZ et al., 2000). Esta informação agora é ampliada para os inseticidas neonicotinóides imidacloprid e thiamethoxan, além do etofenprox e spinosad, que demonstraram ser eficientes no controle de adultos tanto via contato, como ingestão, com maior efeito através da ingestão (Tabela 1). Considerando que o ideal seria dispor de produtos que controlassem a praga através dos três modos de ação (contato, ingestão e profundidade), como ocorre com os inseticidas fosforados, não foi possível selecionar produtos com tais características. O maior potencial de emprego dos novos compostos parece ser na forma de isca-tóxica, em substituição aos fosforados que apresentam sérias restrições de toxicidade (EPA, 2003), o que já reduziria o volume de fosforados aplicados anualmente nos pomares.

$O$ efeito de contato dos inseticidas etofenprox, imidacloprid, spinosad e thiamethoxan não deve ser descartado, pois em condições de campo, principalmente no período de pré-colheita, quando uma carência reduzida é fundamental, existe potencial de emprego destes produtos, visando ao controle de adultos antes da oviposição nos frutos.

Embora os experimentos tenham sido conduzidos em laboratório, acredita-se que as doses que proporcionaram maiores percentuais de mortalidade dos adultos servirão de base para a avaliação nos pomares comerciais, fato que ainda necessita ser realizado, visando viabilizar o emprego destes produtos em fruteiras temperadas.

\section{CONCLUSÕES}

Os inseticidas fenthion e trichlorfom são eficientes no controle de adultos e larvas de Anastrepha fraterculus. $\mathrm{O}$ inseticida benzoato de emamectina não é eficiente no controle de adultos de A. fraterculus via contato e ingestão. Os inseticidas etofenprox, imidacloprid, spinosad e thiametoxam são eficientes no controle de adultos de $\boldsymbol{A}$. fraterculus via contato e ingestão. $\mathrm{O}$ inseticida thiacloprid é eficiente no controle de adultos de $\boldsymbol{A}$. fraterculus, via ingestão. Os inseticidas benzoato de emamectina, etofemprox, imidacloprid, spinosad, thiacloprid e thiamethoxan não causam mortalidade significativa de larvas de $\boldsymbol{A}$. fraterculus no interior de maçãs.

\section{REFERÊNCIAS BIBLIOGRÁFICAS}

ABBOTT, W.S. A method of computing the effectiveness of an insecticide. Journal of Economic Entomology, Lanham, v.18, n.1, p.265-267, 1925.

ADAN, A. et al. Laboratory evaluation of the novel naturally derived compound spinosad against Ceratitis capitata. Pesticide Science, Inglaterra, v.48, p.261-268, 1996.

BLEICHER, J. et al. A mosca-das-frutas em macieira e pessegueiro. Florianópolis, SC : EMPASC, 1982. 28p. (EMPASC. Boletim Técnico, 19).

CALKINS, C.O.; MALAVASI, A. Biology and control of fruit flies (Anastrepha) in Tropical and temperate fruit. Revista Brasileira de Fruticultura, Cruz das Almas, v.17, p.36-45, 1995.

CRUZ, I.B.M. et al. Ocurrence of polytene chromosomes in Anastrepha fraterculus (Diptera: Tephritidae). Cytobios, Cambridge, v. 79, p.45-50, 1994. 
CRUZ, I.B.M. et al. Toxity of fenthiom to Anastrepha fraterculus (Wied.) (Diptera: Tephritidae): dose response analyses. Anais da Sociedade Entomológica do Brasil, Londrina, v.26, p.471479, 1997.

CRUZ, I.B.M. et al. Estudos toxicológicos. In: MALAVASI, A.; ZUCCHI, R.A. (Ed.). Moscas-das-frutas de importância econômica no Brasil: conhecimento básico e aplicado. Ribeirão Preto : Holos, 2000. Cap.8, p.143-150.

ENVIRONMENTAL PROTECTION AGENCY. Food and quality protection act (FQPA) of 1996. Capturado em 06 jan. 2003. Online. Disponível em <http://www.epa. gov/opppsps $1 /$ fqpa/>.

FACHINELLO, J.C. Situação e perspectiva da produção integrada na Europa In: PROTAS, J.F.S.; SANHUEZA, R.M.V. Produção integrada de frutas: o caso da maça no Brasil. Bento Gonçalves : Embrapa Uva e Vinho, 2003. p.21-32. (EMBRAPA: Circular Técnica, 34).

FURTADO, D. Sisvar versão 4.3 (Build 4.5). Lavras : Universidade Federal de Lavras, 2003. Capturado em 10 jan. 2003. Online. Disponível na Internet: < $\underline{\text { http:// }}$ www.ufl.br/>.

KING, J.R.; HENNESSEY, M.K. Spinosad bait for the Caribbean fruit fly (Diptera: Tephritidae). Florida Entomology, Flórida, v.79, p.526-531, 1996.

KOVALESKI, A. et al. Controle químico em macieiras. In: MALAVASI, A.; ZUCCHI, R.A. (Ed.). Moscas-das-frutas de importância econômica no Brasil: conhecimento básico e aplicado. Ribeirão Preto : Holos, 2000. Cap.17, p.135-141.

KOVALESKI, A.; RIBEIRO, L.G. Manejo de pragas na produção integrada de maçãs. In: PROTAS, J.F.S.; SANHUEZA, R.M.V. Produção integrada de frutas: o caso da maça no Brasil. Bento Gonçalves : Embrapa Uva e Vinho, 2003. p.61-76. (EMBRAPA: Circular Técnica, 34).
LORENZATO, D. et al. Competição de inseticidas, doses e épocas visando a recuperação e proteção de maçãs frente ao ataque de mosca-das-frutas na cultura da macieira (Malus domestica Bock). Agronomia Sulriograndense, Porto Alegre, v. 22 , p. $89-101,1986$

NORA, I. et al. Ocorrência de moscas-das-frutas em Santa Catarina. In: MALAVASI, A.; ZUCCHI, R.A. (Ed.). Moscas-das-frutas de importância econômica no Brasil: conhecimento básico e aplicado. Ribeirão Preto : Holos, 2000. Cap.40, p.271-275.

PUZZI, D.; ORLANDO, A. Ensaios de combate às "moscasdas-frutas" Ceratitis capitata (Wied.) e Anastrepha spp. por meio de pulverizações de iscas envenenadas. O Biológico, São Paulo, v.23, p.21-25, 1957.

REIS FILHO, W. Controle químico da mosca-das-frutas Anastrepha fraterculus (Wied., 1830) (Diptera: Tephritidae) em macieira. Revista Brasileira de Fruticultura, Cruz das Almas, v.16, p.64-69, 1994.

SALLES, L.A.B. Bioecologia e controle da mosca-das-frutas sul-americana. Pelotas : Embrapa-CPACT, 1995a. 58p.

SALLES, L.A.B. Isca tóxica para o controle de adultos de Anastrepha fraterculus (Wied.) (Diptera: Tephritidae). Anais da Sociedade Entomológica do Brasil, Londrina, v.24, p.153$157,1995 \mathrm{~b}$

SALLES, L.A.B. de. Principais pragas e seu controle. In: MEDEIROS, C.A.B.; RASEIRA, M. do C. A cultura do pessegueiro. Brasília : EMBRAPA- CPACT, 1998. Cap.8, p.206-242.

SALLES, L.A.B. Biologia e ciclo de vida. In: MALAVASI, A.; ZUCCHI, R.A. (Ed.). Moscas-das-frutas de importância econômica no Brasil: conhecimento básico e aplicado. Ribeirão Preto : Holos, 2000. V.1, cap.8, p.81-86.

SALLES, L.A.B.; KOVALESKI, A. Inseticidas para controle da mosca-das-frutas. Horti Sul, Pelotas, v.1, p.10-11, 1990. 